Research Paper

\title{
RANKL Reduces Body Weight and Food Intake via the Modulation of Hypothalamic NPY/CART Expression
}

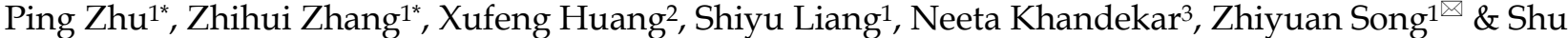 \\ $\operatorname{Lin}^{1,2}$ \\ 1. Department of Cardiology, Southwest Hospital, Third Military Medical University (Army Medical University), China \\ 2. School of Medicine, University of Wollongong and Illawarra Health and Medical Research Institute, NSW 2522, Australia \\ 3. Neurological Diseases Division, Research Program, Garvan Institute of Medical Research, 384 Victoria Street, Darlinghurst, Sydney, NSW 2010, Australia \\ * These authors contributed equally to this work. \\ $\square$ Corresponding authors: Prof. Shu Lin, No.30 Gaotanyan, Shapingba, Chongqing, 400038, China. Phone: 86 15683713870; E-mail: shulin1956@126.com and \\ Prof. Zhiyuan Song, No.30 Gaotanyan, Shapingba, Chongqing, 400038, China. Phone: 86 13908327066; E-mail: zysong2010@126.com \\ (c) Ivyspring International Publisher. This is an open access article distributed under the terms of the Creative Commons Attribution (CC BY-NC) license \\ (https://creativecommons.org/licenses/by-nc/4.0/). See http://ivyspring.com/terms for full terms and conditions.
}

Received: 2017.12.15; Accepted: 2018.05.31; Published: 2018.07.01

\begin{abstract}
The receptor activator of nuclear factor- $\mathrm{K} B$ ligand (RANKL) modulates energy metabolism. However, how RANKL regulates energy homeostasis is still not clear. This study aims to investigate the central mechanisms by which central administration of RANKL inhibits food intake and causes weight loss in mice. We carried out a systematic and in-depth analysis of the neuronal pathways by which RANKL mediates catabolic effects. After intracerebroventricle (i.c.v.) injection of RANKL, the expression of neuropeptide Y (NPY) mRNA in the Arc was significantly decreased, while the CART mRNA expression dramatically increased in the Arc and DMH. However, the agouti-related protein (AgRP) and pro-opiomelanocortin (POMC) mRNA had no significant changes compared with control groups. Together, the results suggest that central administration of RANKL reduces food intake and causes weight loss via modulating the hypothalamic NPY/CART pathways.
\end{abstract}

Key words: Receptor activator of NF-кB ligand; Food intake; Cocaine- and amphetamine-regulated transcript; Neuropeptide Y; Hypothalamus

\section{Introduction}

About 500 million adults have developed obesity worldwide, which is associated with a greater risk of type II diabetes and cardiovascular disease that makes obesity as a major health, social and economic problems to most countries [1-3]. Various brain nuclei are involved in the control of body metabolisms, including insulin production and energy expenditure $[4,5]$. Recent studies have proved a link between the central RANKL/RANK and energy homeostasis $[6,7]$. RANKL is a 317-amino acid peptide that belongs to tumour necrosis factor (TNF) cytokine family [8]. Two forms of RANKL have been found, a membrane-bound molecule expressed on osteoblasts and a soluble form. RANKL plays an important role in bone reconstruction by binding and activating its receptor RANK, a 616-amino acid peptide $[9,10]$. RANKL/RANK protein and mRNA are expressed in bone and bone marrow, lymphoid tissues [11], the hypothalamus and septal regions of the brain [6,7]. Importantly, elevated levels of circulating soluble RANKL have been observed in the circulation of patients with anorexia nervosa compared to healthy, age-matched controls [12] and it is worth noting that RANKL levels depend on the severity of the anorexia nervosa [13]. Similarly, mice intraperitoneally injected with an adenovirus vector harbouring murine soluble RANKL cDNA exhibit reduced food intake and body weight [14]. Together, these findings support anorectic effects of RANKL. However, the precise hypothalamic nuclei and neuropeptides that mediate the effects of RANKL remain unexplored.

Arcuate (Arc) and dorsomedial (DMH) nuclei in the hypothalamus are two main areas regulating energy and appetite. These nuclei in the Arc 
interchange and integrate peripheral signals like calories intake, nutritional status to regulate appetite and energy expenditure $[4,15,16]$. Previous studies have shown a link between the $\mathrm{DMH}$, ingestion and body weight regulation [17]. Lesion of the DMH results in hypophagia, reduced body weight and impaired growth in rats [18]. In the Arc, two types of neurons, NPY/agouti-related protein (AgRP) neurons and CART/pro-opiomelanocortin (POMC), are main functional units to produce orexigenic or anorexigenic neurotransmitters respectively $[19,20]$. NPY is a 36-amino acid peptide that drives body weight gain, increased food intake and decreased energy expenditure [4, 21, 22], while CART causes loss of appetite. Studies on bone remodelling reveal that RANKL signalling is modulated by CART and NPY. For instance, homozygous deletion of CART results in increased RANKL expression in bone and decreased bone mass [23], while NPY inhibits RANKL expression on osteoblasts [24]. However, the relationship among CART, NPY and RANKL in the regulation of energy balance remains to be addressed. A major goal of this study is to explore whether RANKL reduces food intake and causes body weight loss via modulating the hypothalamic NPY/CART neuronal pathways.

\section{Materials and Methods}

\section{Ethical and animal care}

The experimental protocol was approved by the Third Military Medical University Animal Care Committee according to the National Institutes of Health Guide for the Care and Use of Laboratory Animals (NIH publication number 8023). All mice were housed under conditions of controlled temperature $\left(22^{\circ} \mathrm{C}\right)$ and illumination (12-hour light cycle, lights on at 07:00 am) with ad libitum access to water and normal chow (6\% calories from fat, $21 \%$ calories from protein, $71 \%$ calories from carbohydrate, Gordon's Specialty Stock Feeds, Australia) unless otherwise stated.

\section{Body weight, food intake measurement}

Eleven C57BL/6J male mice were divided into two groups, treated with either RANKL (intracerebroventricle, i.c.v. injection, $\mathrm{n}=6$ ) or saline as control group $(\mathrm{n}=5)$. After three days of acclimatization, we implanted ALZET ${ }^{\circledR}$ Micro-Osmotic Pump (LOT NO. 10239-10, Model 1007D, Flow rate $0.5 \mu \mathrm{l}$ /hour) in mice for RANKL i.c.v. injection which contained RANKL (7 days) and allowed for a constant stream of $10 \mathrm{ng}$ per day RANKL to be delivered directly into the third ventricle of brain. The procedure of the
Micro-Osmotic Pump implantation was performed as described in previous study [25]. Meanwhile, we applied the same surgery to the other group, in which pump contained saline as controls. The subsequent measurement of body weight was taken daily at the same time. At the $8^{\text {th }}$ day, cumulative food intake in 24 hours (from $7^{\text {th }}$ day 10:00 to $8^{\text {th }}$ day 10:00) of two groups was calculated separately. Both groups of mice were used for the detection of in situ hybridization mRNA expression.

\section{Immunohistologic analysis of RANKL altered c-fos expression in brain}

At the age of 16 weeks, ten C57BL/6J male mice were divided into two groups: one group of mice for RANKL i.v. injection $(n=5)$ and the other group of mice for saline i.v. injection as controls $(\mathrm{n}=5)$, and both groups were used for c-fos detection. The mice were i.v. injected with $10 \mu \mathrm{g}$ RANKL diluted in $1 \mathrm{ml}$ saline or $1 \mathrm{ml}$ saline for $30 \mathrm{~min}$ and then deeply anesthetized with ketamine-xylazine $(100 \mathrm{mg} / \mathrm{kg}$ and $20 \mathrm{mg} / \mathrm{kg}$ from Parke Davis Pfizer, Sydney, Australia and Bayer AG, Leverkusen, Germany, respectively) through intraperitoneally injection. From the left heart ventricle, $25 \mathrm{ml}$ of phosphate buffered saline (PBS) and $4 \%$ paraformaldehyde dissolved in PBS were perfused into the whole body successively. After dislocating and sacrificing the mice, we removed the brain immediately, then placed it in $4 \%$ paraformaldehyde PBS solution for 30 minutes and transferred to $30 \%$ sucrose solution to remain overnight and restored in $-70{ }^{\circ} \mathrm{C}$ refrigerator. $30 \mathrm{~mm}$ thickness of coronal slices were placed in PBS and washed in $50 \%$ ethanol which contained $1 \% \mathrm{H}_{2} \mathrm{O}_{2}$ for 20 minutes to abolish endogenous peroxidase activity. Brain section was incubated with the primary antibody, rabbit anti-mouse c-fos protein (Santa Cruz Biotechnology Inc, Santa Cruz, CA, USA) which was diluted at 1: 4000 in PBS containing 0.1\% TRITON $\mathrm{X}-100$, at room temperature overnight. After washing in $0.1 \%$ TRITON X-100-PBS for 10 minutes and repeated three times, sections were incubated for 3 hours with the biotinylated secondary antibody (Sigma-Aldrich, St Louis, MO, USA), diluted at 1:250 in PBS. Again, washing in PBS for 10 minutes 3 times, brain sections were incubated with Avidin Biotin-Peroxidase VectastainH (Vector Laboratories, Burlingame, CA, USA) at room temperature for 30 minutes. Then sections were rinsed in PBS and treated with diaminobenzidine (Dako, Carpinteria, CA, USA) for 5 minutes. Finally, sections were rinsed with water, mounted on slides, and dehydrated before cover slipping. Sections were visualized for c-fos-like immunoreactivity by using a Zeiss Axiophot microscope equipped with the Prog Res digital 
camera (Carl Zeiss Imaging Solutions $\mathrm{GmbH}$, Munich, Germany). Semiquantitative analysis of c-fos has been described previously [4].

\section{Double labelling of c-fos and NPY}

In order to verify whether NPY neurons in the hypothalamus are involved in response to RANKL i.v. injection, double-labelling experiment was performed to ascertain whether NPY neurons were activated by RANKL i.v. injection. At the age of 16 weeks, eight transgenic NPY Green Fluorescent Protein (GFP) male mice expressing green fluorescent protein (purchased from Jackson Laboratory) were divided into two groups: one group of mice for RANKL i.v. injection $(n=4)$ and the other group of mice for saline i.v. injection as controls $(n=4)$, and both group of mice were used for c-fos detection. These NPYGFP mice were i.v. injected with $10 \mu \mathrm{g}$ RANKL diluted in $1 \mathrm{ml}$ saline or $1 \mathrm{ml}$ saline for $30 \mathrm{~min}$ and then sacrificed after deeply anaesthetized. Following steps were carried out as c-fos immunohistochemistry test stated above. Brain section was incubated with the primary antibody, rabbit anti-mouse c-fos protein (Santa Cruz Biotechnology Inc, Santa Cruz, CA, USA) diluted at 1:4000. The secondary antibody against c-fos visualizing red fluorescent was Alexa Fluor 594 goat anti-rabbit IgG (A11037, Life Technologies, Canada) diluted at 1:250. Sections were mounted with fluoromount and quantified for c-fos immunoreactivity in NPY-GFP transgenic mice using a ProgRes 3008 camera (Zeiss, Jena, Germany).

\section{Double labelling of $c$-fos and CART mRNA}

In order to determine whether CART neurons in the hypothalamus are activated by RANKL i.v. injection, four C57BL/6J male mice, sixteen week old, were i.v. injected with $10 \mu \mathrm{g}$ RANKL diluted in $1 \mathrm{ml}$ saline, and another four mice were i.v. injected with $1 \mathrm{ml}$ saline as controls. At $30 \mathrm{~min}$ after treatment, mice were deeply anaesthetised, and the brains were fixed by perfused with $25 \mathrm{ml}$ phosphate buffered saline (PBS) and 4\% paraformaldehyde dissolved in PBS. After soaking in $30 \%$ sucrose solution overnight, the brain was cut into coronal sections of $30 \mu \mathrm{m}$ thickness. Immunoreactivities of c-fos were carried out as stated above. Brain sections were incubated with secondary antibodies, goat anti-rabbit (Sigma-Aldrich, St Louis, MO, USA), for three hours, which was diluted at 1:250 in PBS. Sections were mounted and CART in situ hybridisation was performed as previously described [26]. DNA oligonucleotides complementary to mouse CART (5'-TCCTTCTCGTGGGACGCATCATCCACG GCAGAGTAGATGTCC AGG-3') was labelled with [35'] thio-dATP. Co-localization of c-fos and CART mRNA were captured and counted under a Zeiss Axiophot microscope.
In Situ Hybridization for Quantification of NPY, CART, POMC and AgRP mRNA Expression

At the $8^{\text {th }}$ day, all mice treated with RANKL $(n=6)$ or saline $(n=5)$ from implanted micro-osmotic pump were deeply anesthetized with ketamine-xylazine (100 mg / kg and $20 \mathrm{mg} / \mathrm{kg}$ from Parke Davis Pfizer, Sydney, Australia and Bayer AG, Leverkusen, Germany, respectively) through intraperitoneal injection. After sacrificing mice with the same procedure stated above, we cut $20 \mu \mathrm{m}$ coronal slices and prepared them as described in previous study $[26,27]$. For radioactive in situ hybridization, DNA oligonucleotides complementary to mouse NPY (5'-GAGGGTCAGTCCACACAGCCCCATTCGCTTG TTACCTAGCAT-3') CART (5'-TCCTTCTCGTGGGA CGCATCATCCACGGCAGAGTAGATGTCCA

GG-3'), POMC (5'-TGGCTGCTCTCCAGGCACCAG CTCCACACATCTATGGAG G-3'), or AgRP (5'-AGC TTGCGGCAGTAGCAAAAGGCATTGAAGAAGCG GCA GTAGCAC-3') were labelled with [35'] thio-dATP (Amersham Biosciences, Little Chalfont, Buckinghamshire, UK) using terminal deoxynucleotidyl transferase (Roche, Mannheim, Germany). The mRNA levels of NPY, CART, POMC and AgRP in the Arc, and CART in the DMH were evaluated, respectively, by measuring silver grain densities over individual neurons from photo-emulsion dipped sections, as described previously $[4,26]$.

\section{Statistical analysis}

All statistical analyses were performed using GraphPad Prism Version 6.0 (GraphPad Software, Inc). Differences between means were assessed, as appropriate, by two- or one-way ANOVA followed by Bonferroni post hoc analysis. For all statistical analyses, a $\mathrm{P}$ value $<0.05$ was considered to be statistically significant. Data were presented as means \pm SEM.

\section{Results}

\section{Body weight and food intake in response to RANKL administration}

During 7 days of treatment, the mice treated with RANKL displayed a significant reduction of body weight compared with controls (Fig. 1A). Average food intake of each mouse in 24 hours measured at day $8^{\text {th }}$ showed that the mice treated with RANKL had lower food intake compared with controls, which demonstrated that the administration of central RANKL inhibited food intake (Fig. 1B). 

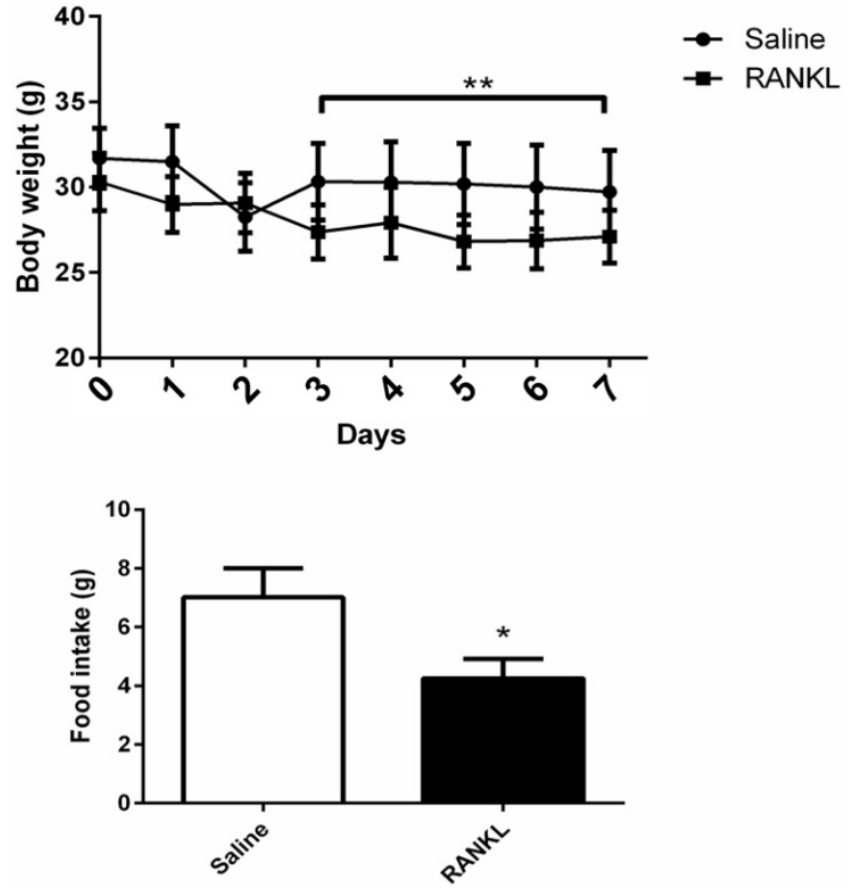

Figure 1. The body weight and food intake of mice altered after RANKL injection. Quantifications of body weight and food intake. Data are mean \pm SEM. $n=6$ in RANKL group, $n=5$ in Saline group $* * \quad p<0.001$ compared with Saline group. $*_{\mathrm{p}}<0.05$ compared with Saline group.

\section{Activated c-fos immunoreactive neurons in hypothalamus after RANKL administration}

To clarify which regions of hypothalamus have been influenced by i.v injection of RANKL, we examined c-fos immunohistochemistry (Fig. 2). The c-fos, a proto-oncogene which is referred to as an immediate early gene for c-fos mRNA and protein, is generally among the first to be expressed. We observed that there were significantly more marked neurons in the section of RANKL treated group than control group in the Arc (Fig. 2A-C) and DMH (Fig. 2D-F) regions. The elevated expression of c-fos suggested that neurons in these regions were activated by RANKL, and revealed that RANKL might induce changes in NPY and its downstream signalling pathways.

\section{NPY and CART mRNA co-localised with c-fos neurons}

Considering that administration of peripheral RANKL triggered more expression of c-fos gene in the Arc and DMH, we detected some neuropeptide genes that regulated energy homeostasis to show which genes were activated on neurons labelled by c-fos after i.v injection of RANKL. In the Arc, we found significant $54 \%$ overlap in expression of NPYGFP neurons (Fig. 3B) and c-fos (Fig. 3A) at 30 min after i.v. administration of RANKL (Table. 1) (Fig. 3C), which suggested that NPY-producing cells may be involved in mediating RANKL-induced decreased appetite and changes in energy intake. In addition, the double-labelling method combining c-fos immunohistochemistry with in situ hybridization showed that about $72 \%$ neurons expressed c-fos immunoreaction and CART mRNA in the DMH (Table. 1) (Fig. 4A-B). The expression of CART mRNA at $30 \mathrm{~min}$ after $i . v$ injection of RANKL indicated that DMH CART neurons were also critical in mediating anorectic effect of RANKL.
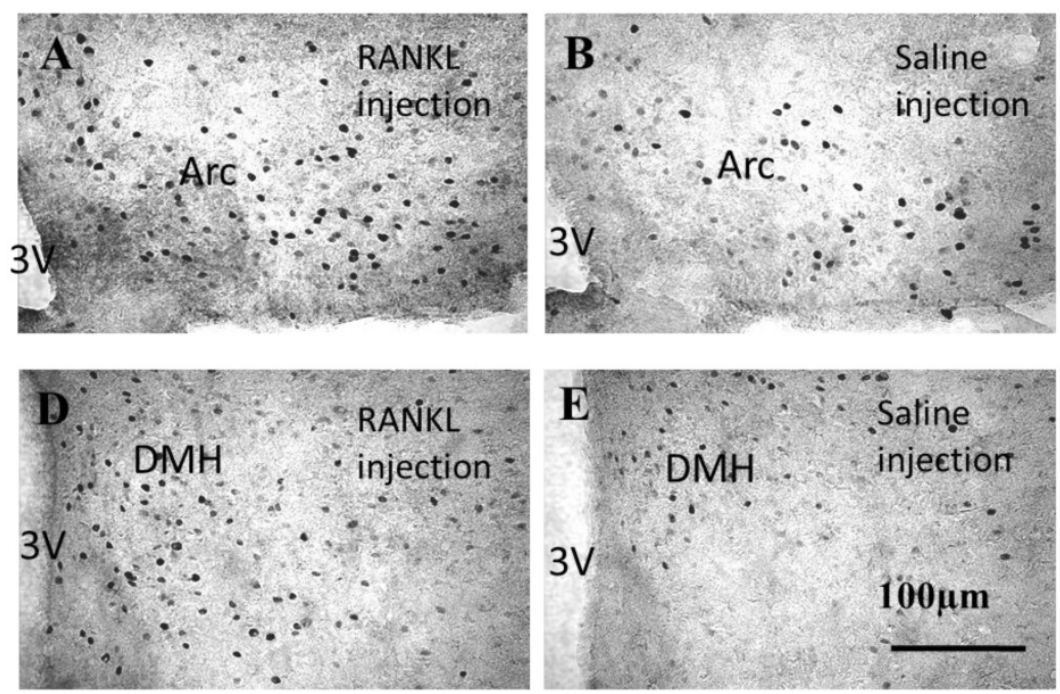
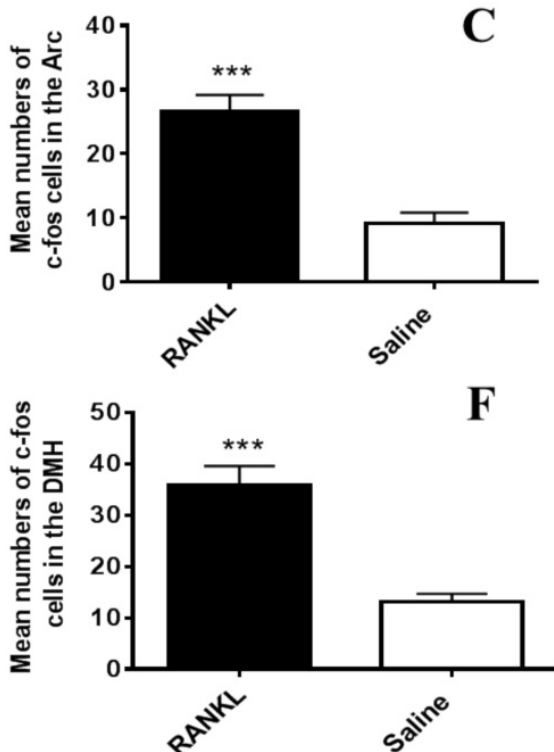

Figure 2. Activated c-fos immunoreactive neurons in the Arc and DMH after RANKL administration. A-F. Immunohistochemical analysis of c-fos expression in the Arc and DMH after i.v administration of RANKL or saline. The c-fos positive neurons were sharply increased in the $A r c$ (A-C) and DMH (D-F) of RANKL peripheral injection. Scale bar $100 \mu \mathrm{m}$. Quantification of c-fos neurons expressed in the Arc and DMH. Data are mean \pm SEM of 5 mice each group. *** $p<0.001$ vs Saline controls. $3 \mathrm{~V}$ : the third cerebral ventricle. Arc: Arcuate nucleus. 

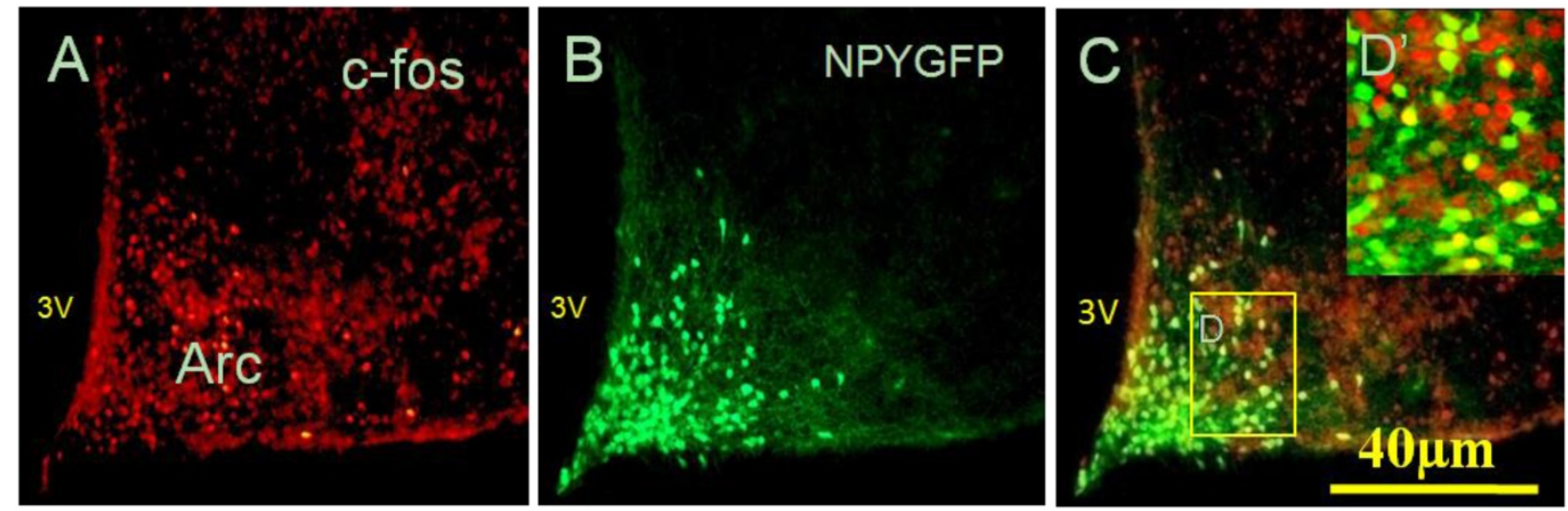

Figure 3. NPY co-localised with c-fos neurons in response to RANKL i.v injection. Fluorescent immunohistochemical identification of c-fos and NPY GFP neurons co-expressed in the Arc, 30 min after RANKL i.v injection. Red staining showed c-fos positive neurons only (A), with green staining indicating NPY neurons expressing GFP (B),

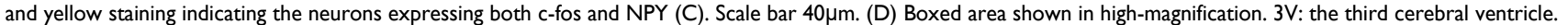

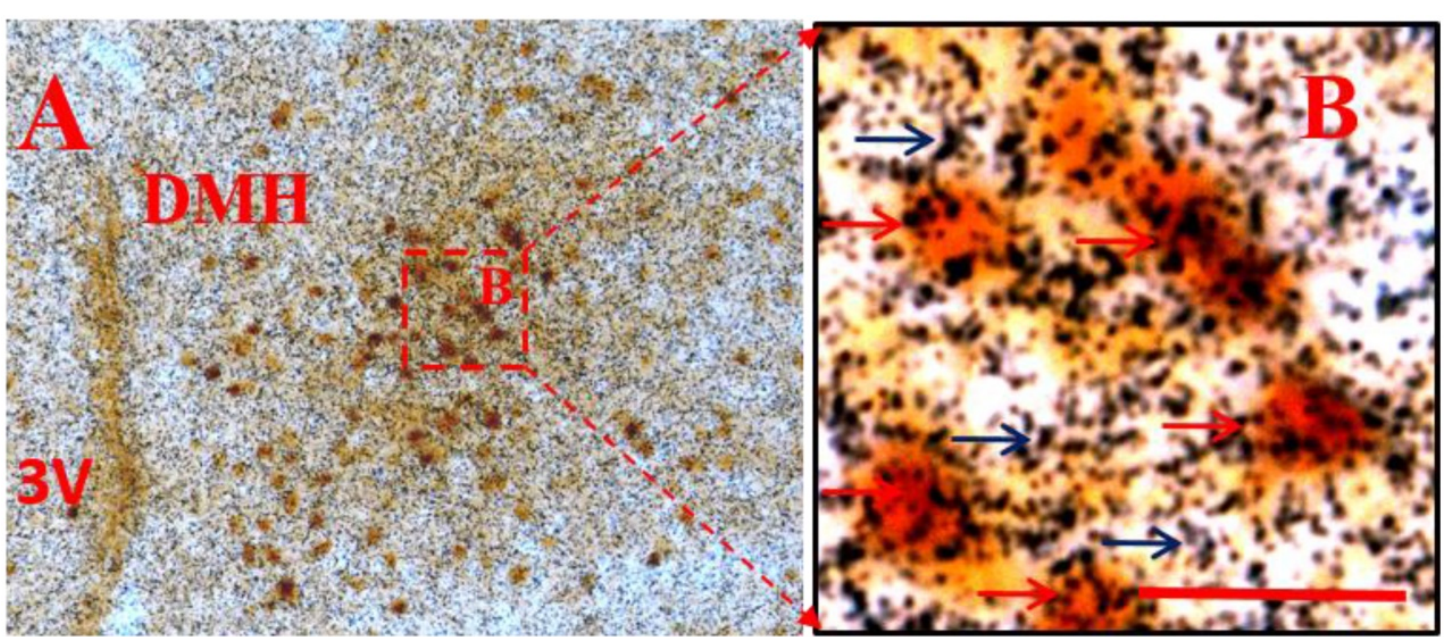

Figure 4. CART mRNA co-localised with c-fos neurons in the DMH after i.v. injection of RANKL. Double labeling of in situ hybridization for CART mRNA and immunohistochemistry for c-fos expression in the DMH, 30 min after i.v. injection of RANKL. A: low-magnification. B: boxed area shown in high-magnification. Black arrows indicate CART mRNA and red arrows indicate double-labelled c-fos and CART mRNA. 3V: the third ventricle. Scale bar: $B=5 \mu m$.

Table 1. NPY and CART mRNA co-localised with c-fos neurons

\begin{tabular}{llll}
\hline & GFP-positive cells & GFP-positive cells expressing c-fos & \% GFP-positive cells expressing c-fos \\
NPY-GFP mice $(\mathrm{n}=4)$ & $89.1 \pm 4.2$ & $47.8 \pm 3.7$ & $54.5 \pm 6.7$ \\
& c-fos positive cells & c-fos positive cells expressing CART mRNA & $\%$ c-fos positive cells expressing CART mRNA \\
C57BL/6J mice $(\mathrm{n}=4)$ & $42.5 \pm 4.7$ & $30.3 \pm 3.5$ & $72.5 \pm 6.5$ \\
\hline
\end{tabular}

Data represent mean \pm SEM.

The expression of NPY, CART, POMC and AgRP mRNAs in response to central administration of RANKL

Through c-fos immunoreactivity test, we elucidated that the Arc and DMH were influenced by RANKL. Given that both the regions are closely related to energy homeostasis, we detected the expression of several neurotransmitters which modulated energy metabolism through in situ hybridization. Compared with control group, RANKL treated group displayed significant decreased expression of NPY mRNA in the Arc (Fig. 5A-C), whereas the expression of CART mRNA in the Arc of RANKL treated group was significantly increased
(Fig. 5D-F). In addition, no significant changes of POMC mRNA were detected (Fig. 6A-C), even though POMC was co-expressed with CART [28]. As we know, AgRP is co-expressed with NPY and capable of increasing appetite and decreasing metabolism and energy expenditure, but there was no significant decrease of AgRP mRNA expression in the Arc of RANKL treated group compared with control group (Fig. 6D-F). In the DMH, there was up-regulation of CART mRNA expression in RANKL group (Fig. 7A-C). These experiments revealed that the central injection of RANKL may modulate the expression of NPY mRNA in the Arc and CART mRNA in the Arc and DMH to inhibit food intake and reduce body weight. 

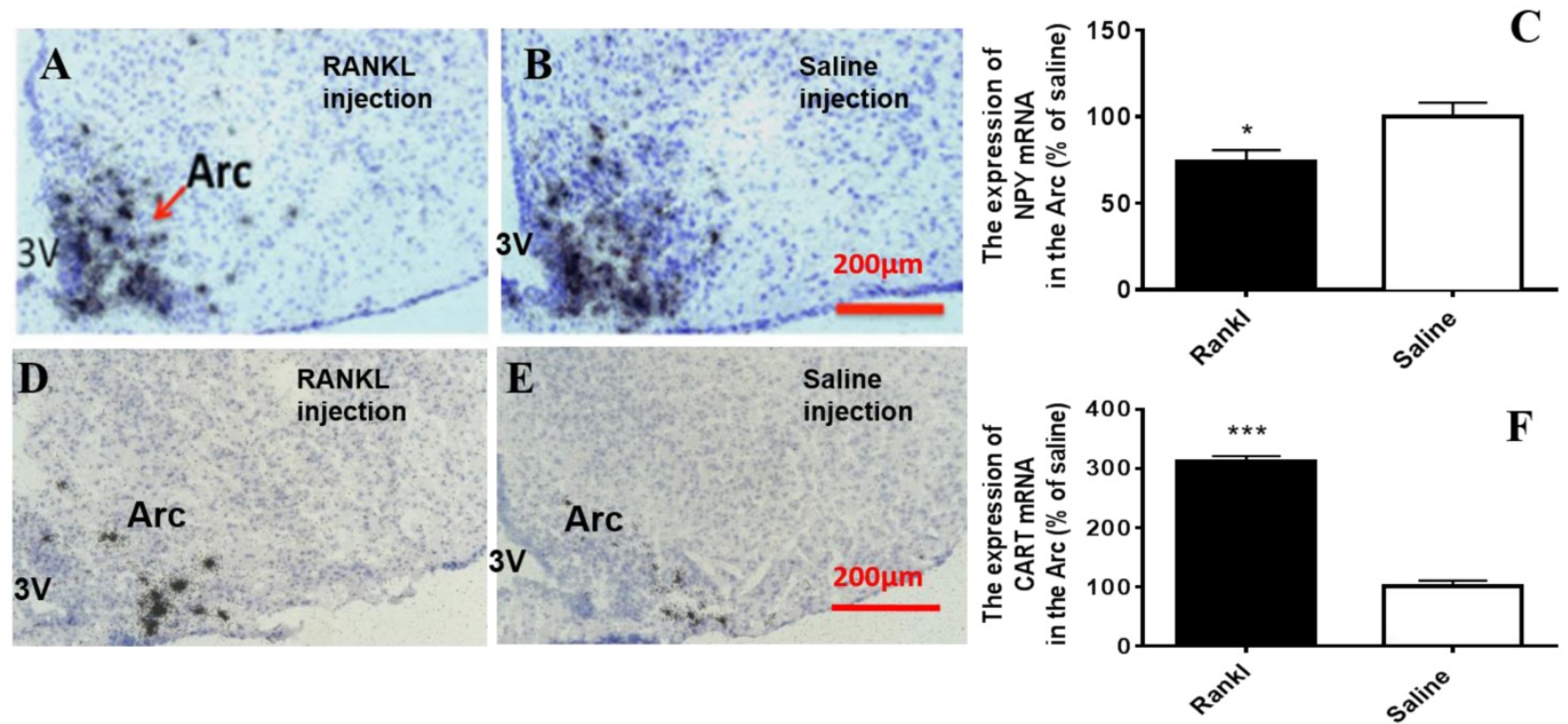

Figure 5. The RANKL influenced the expression of NPY mRNA and CART mRNA in the Arc. Bright filed micrographs of in situ hybridisation showed NPY mRNA in the Arc, 7 days after i.c.v. injection of RANKL (A) or saline (B). Quantification of NPY mRNA expressed in the Arc comparing to saline mice (C). Bright filed micrographs of in situ hybridisation showed CART mRNA in the DMH, 7 days after i.c.v. injection of RANKL (D) or saline (E). Quantification of CART mRNA expressed in the Arc comparing to saline mice $(F)$. Scale bar $200 \mu \mathrm{m}$. Data were mean \pm SEM of $5-6$ mice each group. ${ }^{* * *} p<0.001$ vs Saline controls, ${ }^{*} p<0.05$ vs. Saline controls. $3 V$ : the third ventricle. Arc: Arcuate nucleus
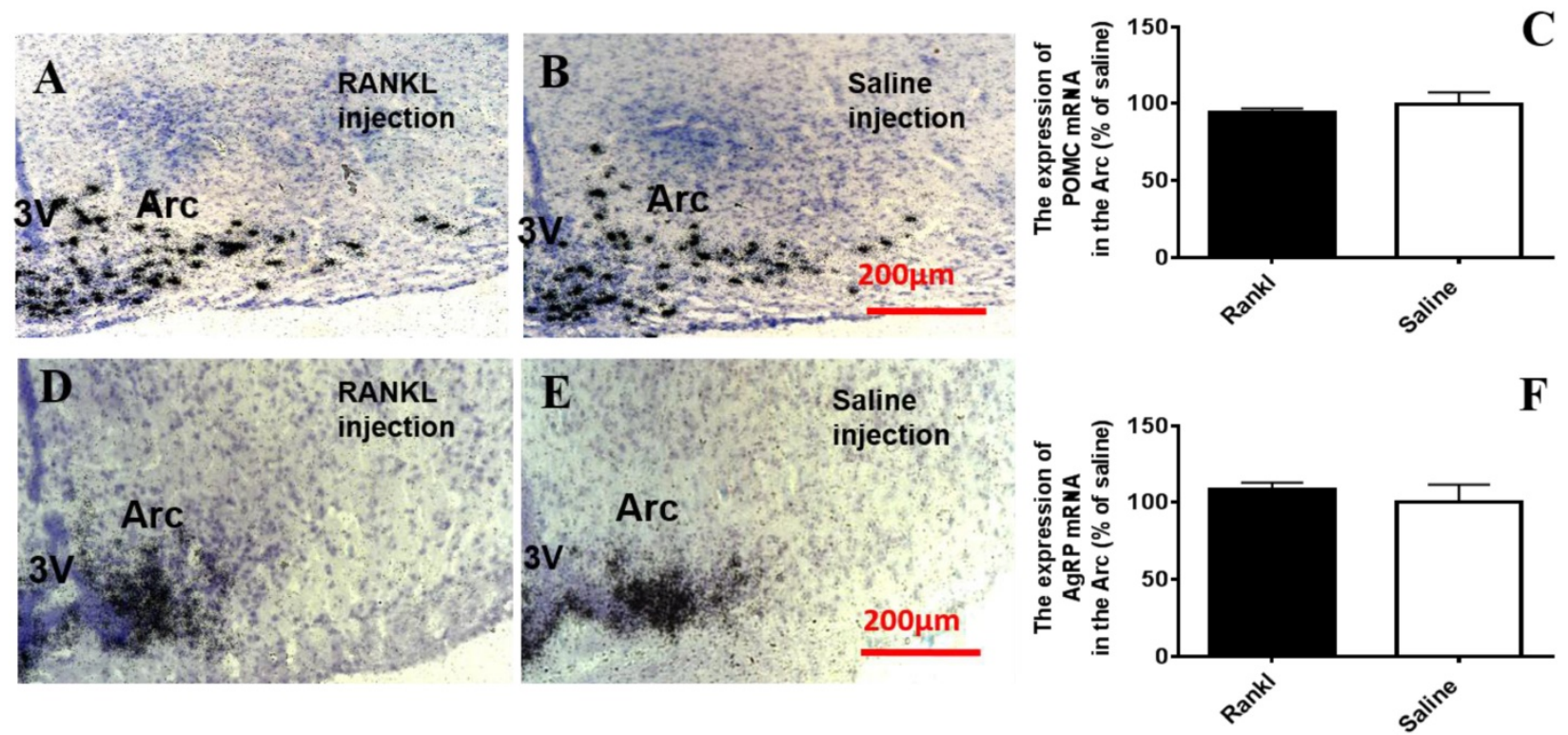

Figure 6. The RANKL did not affect POMC mRNA and AgRP mRNA expression in the Arc. Bright filed micrographs of in situ hybridisation showed POMC mRNA in the Arc 7 days after i.c.v. injection of RANKL (A) or saline (B). Scale bar 200um. Quantification of POMC mRNA expressed in the Arc as a percent of saline mice (C). Bright filed micrographs of in situ hybridisation showed AgRP mRNA in the Arc, 7 days after i.c.v. injection of RANKL (D) or saline (E). Scale bar 200 $\mu m$. Quantification of CART mRNA expressing in the Arc as a percent of saline mice (F). Data were mean \pm SEM of $5-6$ mice each group. $3 \mathrm{~V}$ : the third ventricle. Arc: Arcuate nucleus

C
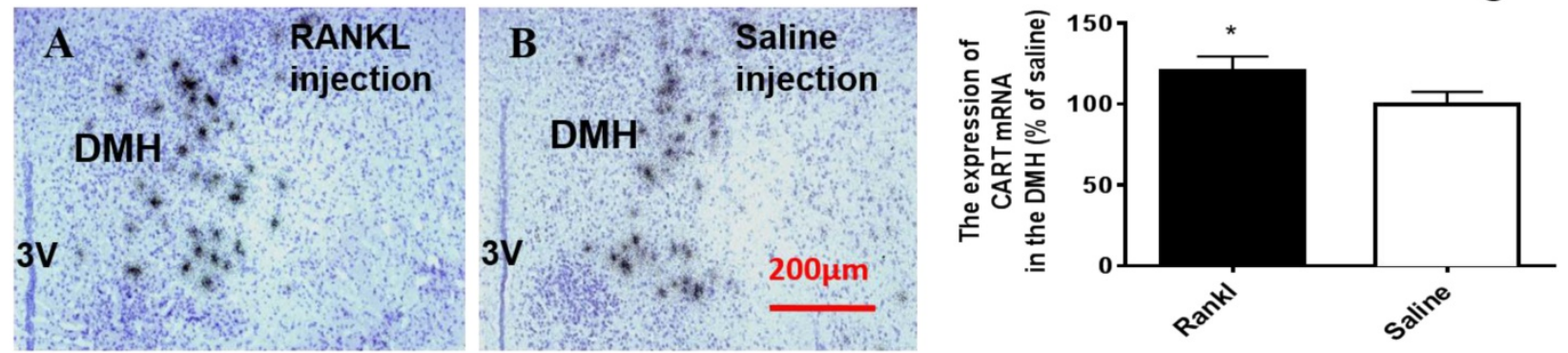

Figure 7. The RANKL influenced the expression of CART mRNA in the DMH. Bright filed micrographs of in situ hybridisation showed CART mRNA in the DMH, 7 days after i.v. injection of RANKL (A) or saline (B). Scale bar 100um. Quantification of CART mRNA expressed in the DMH as a percent of saline mice (C). Data are mean \pm SEM of $5-6$ mice each group. ${ }^{* * *} p<0.001$ vs Saline controls, ${ }^{*} p<0.05$ vs Saline controls. $3 \mathrm{~V}$ : the third ventricle. 


\section{Discussion}

Obesity is a chronic metabolic disease, attributing to various factors, such as genetic factors, unhealthy eating patterns, or a combination of these factors [16]. The main pathogenesis of obesity is energy metabolic imbalance and weight regulation disorder. Many neurotransmitters in the hypothalamus have been implicated in the regulation of energy homeostasis and weight regulation [4, 21, 22, 29-31]. In our research, we verified whether NPY, CART, POMC and AGRP were involved in the catabolic effects via RANKL signal pathway.

RANKL, an acid peptide which can pass through the blood brain barrier [32] and binds to RANK expressed in the hypothalamus. RANKL treatment significantly inhibited daily food intake compared with control group, implying that administration of peripheral RANKL led to altered feeding behaviour. C-fos, a proto-oncogene which is referred to as an immediate early gene for c-fos mRNA and protein, is generally among the first to be expressed after specific stimulation. We identified changes in hypothalamic c-fos expression in response to peripheral RANKL administration. The present study showed there was altered expression of c-fos compared with controls. After i.v. RANKL administration, particularly strong increases in c-fos expression were noted in the Arc and DMH. This suggested that these neurons activated by RANKL may play an important role in inhibiting food intake and decreasing the body weight of mice.

NPY/AgRP neurons in the Arc, modulate energy homeostasis by promoting food intake and reducing energy expenditure [22]. Some circulating hormones and metabolic mediators, such as leptin and RANKL [33], can across the blood brain barrier to reach NPY/AgRP neurons in the Arc. Food deprivation can sharply elevate the NPY levels in the Arc, which is capable of correcting negative energy balance $[4,34]$. I.c.v. delivery of NPY leads to robust hyperphagia and the development of morbid obesity [35], indicating that the NPY not only regulates feeding but also controls energy storage. In current study, after peripheral treatment of RANKL, there was significant overlap in expression of Arc NPY and c-fos labelled neurons in NPY GFP mice. In situ hybridisation showed a down-regulation of NPY mRNA after i.c.v. injection of RANKL. The levels of AgRP mRNA in the Arc remained unchanged in RANKL treated group comparing to saline injection group. All results suggested that reduced NPY diminished appetite, which could be a possible reason to weight loss of mice after administration of RANKL.

As we know, CART is an orexigenic neurotransmitter produced by POMC/CART neurons in the Arc. It also reduces body weight by inhibiting food intake and increasing energy expenditure. Fasting down-regulates CART mRNA in the Arc, PVN and PeF [19]. Moreover, delivery of CART strongly curbed both spontaneous and fastinginduced food intake [36]. In this study, we used double-labelling techniques to determine the chemical nature of neurons activated within hypothalamic areas which were associated with energy metabolism [37]. Approximately $72 \%$ of c-fos neurons in the $\mathrm{DMH}$ were co-localised with CART mRNA after treatment of RANKL, indicating that DMH CART neurons were also critical in mediating anorectic effect of RANKL. The results from in situ hybridisation showed up-regulation of CART mRNA in the Arc and the $\mathrm{DMH}$ after i.c.v. injection of RANKL, and the expression of POMC mRNA remained almost the same in two groups. It was possible that administration of RANKL induced the production of CART, which subsequently led to weight loss.

Furthermore, CART and NPY are reciprocally regulated. NPY-stimulated feeding is blocked by administration of CART [36, 38]. It has been elucidated that Arc NPY neurons project to the DMH, which influences the energy homeostasis [39-41]. In the DMH, the neurons expressing NPY have been found to co-express CART [42]. Moreover, NPY/ AgRP neurons can directly inhibit CART/POMC neurons either by NPY-Y receptor activation or release of GABA [43]. Our findings also indicate that CART in the DMH is the downstream of NPY, which is consistent with previous evidence [21]. Thus, decreased Arc NPY may lead to upregulation of CART in the DMH. In addition, after treatment of RANKL to mice, NPY mRNA was decreased and CART mRNA was increased in the Arc. As two sets of neurons present reciprocal metabolic effects, the changes of mRNA in both neurons result in inhibitory food intake. Moreover, as the downstream of NPY, increased CART in the DMH was regulated either by NPY or by RANKL directly. All these findings elucidate the possibility that RANKL has anti-obesity effects.

In summary, the primary outcomes of this study have provided a new understanding about the neuronal mechanisms underlying the effects of RANKL on food intake and energy homeostasis, which is mediated by the hypothalamic NPY-CART pathway. These results may provide a platform to identify new drug targets for the development of suitable pharmacological treatments for obesity.

\section{Acknowledgements}

This work was supported by National Natural Science Foundation of China (No. 81670402 and No. 
8167020519); the Youth Project of National Natural Science Foundation of China (No. 81300142)

\section{Authorship}

ZHZ and PZ performed this research and drafted the manuscript; SYL and NK assisted to collect data and analyze; XFH revised the manuscript; SL and ZYS designed and revised the paper.

\section{Competing Interests}

The authors have declared that no competing interest exists.

\section{References}

1. Garvey WT, Mechanick JI, Brett EM, Garber AJ, Hurley DL, Jastreboff $\mathrm{AM}$, et al. American Association of Clinical Endocrinologists and American College of Endocrinology Comprehensive Clinical Practice Guidelines for Medical Care of Patients with Obesity. Endocr Pract. 2016; 22 Suppl 3: 1-203.

2. Finucane MM, Stevens GA, Cowan MJ, Danaei G, Lin JK, Paciorek CI, et al. National, regional, and global trends in body-mass index since 1980: systematic analysis of health examination surveys and epidemiological studies with 960 country-years and 9.1 million participants. Lancet. 2011; 377: 557-67.

3. Piepoli MF, Hoes AW, Agewall S, Albus C, Brotons C, Catapano AL, et al. 2016 European Guidelines on cardiovascular disease prevention in clinical practice: The Sixth Joint Task Force of the European Society of Cardiology and Other Societies on Cardiovascular Disease Prevention in Clinical Practice (constituted by representatives of 10 societies and by invited experts)Developed with the special contribution of the European Association for Cardiovascular Prevention \& Rehabilitation (EACPR). European heart journal. 2016; 37: 2315-81.

4. Shi YC, Lau J, Lin Z, Zhang H, Zhai L, Sperk G, et al. Arcuate NPY controls sympathetic output and BAT function via a relay of tyrosine hydroxylase neurons in the PVN. Cell Metab. 2013; 17: 236-48.

5. Yulyaningsih E, Loh K, Lin S, Lau J, Zhang L, Shi Y, et al. Pancreatic polypeptide controls energy homeostasis via Npy6r signaling in the suprachiasmatic nucleus in mice. Cell Metab. 2014; 19: 58-72.

6. Hanada R, Leibbrandt A, Hanada T, Kitaoka S, Furuyashiki T, Fujihara $\mathrm{H}$, et al. Central control of fever and female body temperature by RANKL/RANK. Nature. 2009; 462: 505-9.

7. Kartsogiannis V, Zhou H, Horwood NJ, Thomas RJ, Hards DK, Quinn JM, et al. Localization of RANKL (receptor activator of NF kappa B ligand) mRNA and protein in skeletal and extraskeletal tissues. Bone. 1999; 25: 525-34.

8. Anderson DM, Maraskovsky E, Billingsley WL, Dougall WC, Tometsko ME, Roux ER, et al. A homologue of the TNF receptor and its ligand enhance T-cell growth and dendritic-cell function. Nature. 1997; 390: 175-9.

9. Hanada R, Hanada $\mathrm{T}$, Sigl $\mathrm{V}$, Schramek $\mathrm{D}$, Penninger JM. RANKL/RANK-beyond bones. J Mol Med (Berl). 2011; 89: 647-56.

10. Schoppet $M$, Preissner KT, Hofbauer LC. RANK ligand and osteoprotegerin: paracrine regulators of bone metabolism and vascular function. Arterioscler Thromb Vasc Biol. 2002; 22: 549-53.

11. Nakagawa T, Roth W, Wong P, Nelson A, Farr A, Deussing J, et al. Cathepsin L: critical role in Ii degradation and CD4 T cell selection in the thymus. Science. 1998; 280: 450-3.

12. Ostrowska Z, Ziora K, Oswiecimska J, Swietochowska $\mathrm{E}$ Wolkowska-Pokrywa K. Dehydroepiandrosterone sulfate, osteoprotegerin and its soluble ligand sRANKL and bone metabolism in girls with anorexia nervosa. Postepy Hig Med Dosw (Online). 2012; 66: 655-62.

13. Ostrowska Z, Ziora K, Oswiecimska J, Swietochowska E, Szapska B, Wolkowska-Pokrywa K, et al. RANKL/RANK/OPG system and bone status in females with anorexia nervosa. Bone. 2012; 50: 156-60.

14. Enomoto T, Furuya Y, Tomimori Y, Mori K, Miyazaki J, Yasuda H. Establishment of a new murine model of hypercalcemia with anorexia by overexpression of soluble receptor activator of NF-kappaB ligand using an adenovirus vector. J Bone Miner Metab. 2011; 29: 414-21.

15. Furlong TM, McDowall LM, Horiuchi J, Polson JW, Dampney RA. The effect of air puff stress on c-Fos expression in rat hypothalamus and brainstem: central circuitry mediating sympathoexcitation and baroreflex resetting. Eur J Neurosci. 2014; 39: 1429-38
16. Williams LM. Hypothalamic dysfunction in obesity. Proc Nutr Soc. 2012; 71: 521-33.

17. Bi S. Dorsomedial hypothalamic NPY modulation of adiposity and thermogenesis. Physiology \& behavior. 2013; 121: 56-60.

18. Zheng F, Kim YJ, Chao PT, Bi S. Overexpression of neuropeptide $Y$ in the dorsomedial hypothalamus causes hyperphagia and obesity in rats. Obesity. 2013; 21: 1086-92.

19. Li AJ, Dinh TT, Ritter S. Hyperphagia and obesity produced by arcuate injection of NPY-saporin do not require upregulation of lateral hypothalamic orexigenic peptide genes. Peptides. 2008; 29: 1732-9.

20. Elias CF, Lee CE, Kelly JF, Ahima RS, Kuhar M, Saper CB, et al. Characterization of CART neurons in the rat and human hypothalamus. Comp Neurol. 2001; 432: 1-19.

21. Inui A. Transgenic approach to the study of body weight regulation. Pharmacol Rev. 2000; 52: 35-61.

22. Lin S, Boey D, Herzog H. NPY and Y receptors: lessons from transgenic and knockout models. Neuropeptides. 2004; 38: 189-200.

23. Elefteriou F, Ahn JD, Takeda S, Starbuck M, Yang X, Liu X, et al. Leptin regulation of bone resorption by the sympathetic nervous system and CART. Nature. 2005; 434: 514-20.

24. Teixeira L, Sousa DM, Nunes AF, Sousa MM, Herzog H, Lamghari M. NPY revealed as a critical modulator of osteoblast function in vitro: new insights into the role of Y1 and Y2 receptors. J Cell Biochem. 2009; 107: 908-16.

25. Stayte S, Rentsch P, Li KM, Vissel B. Activin A protects midbrain neurons in the 6-hydroxydopamine mouse model of Parkinson's disease. PloS one. 2015; 10: e0124325.

26. Sainsbury A, Schwarzer C, Couzens M, Herzog H. Y2 receptor deletion attenuates the type 2 diabetic syndrome of ob/ob mice. Diabetes. 2002; 51: 3420-7.

27. Lei M, Schumacher LJ, Lai YC, Juan WT, Yeh $\mathrm{CY}, \mathrm{Wu} \mathrm{P}$, et al Self-organization process in newborn skin organoid formation inspires strategy to restore hair regeneration of adult cells. Proc Natl Acad Sci U S A. 2017; 114: E7101-E10.

28. Caminos JE, Bravo SB, Gonzalez CR, Garces MF, Cepeda LA, Gonzalez $\mathrm{AC}$, et al. Food-intake-regulating-neuropeptides are expressed and regulated through pregnancy and following food restriction in rat placenta. Reprod Biol Endocrinol. 2008; 6: 14

29. Anderson EJ, Cakir I, Carrington SJ, Cone RD, Ghamari-Langroudi M, Gillyard T, et al. 60 YEARS OF POMC: Regulation of feeding and energy homeostasis by alpha-MSH. J Mol Endocrinol. 2016; 56: T157-74.

30. Mavanji V, Perez-Leighton CE, Kotz CM, Billington CJ, Parthasarathy S, Sinton CM, et al. Promotion of Wakefulness and Energy Expenditure by Orexin-A in the Ventrolateral Preoptic Area. Sleep. 2015; 38: 1361-70.

31. Xia T, Cheng Y, Zhang Q, Xiao F, Liu B, Chen S, et al. S6K1 in the central nervous system regulates energy expenditure via MC4R/CRH pathways in response to deprivation of an essential amino acid. Diabetes. 2012; 61: 2461-71.

32. Guerrini MM, Okamoto K, Komatsu N, Sawa S, Danks L, Penninger JM, et al. Inhibition of the TNF Family Cytokine RANKL Prevents Autoimmune Inflammation in the Central Nervous System. Immunity. 2015; 43: 1174-85.

33. Faouzi M, Leshan R, Bjornholm M, Hennessey T, Jones J, Munzberg H. Differential accessibility of circulating leptin to individual hypothalamic sites. Endocrinology. 2007; 148: 5414-23.

34. Sainsbury A, Zhang L. Role of the arcuate nucleus of the hypothalamus in regulation of body weight during energy deficit. Molecular and cellular endocrinology. 2010; 316: 109-19.

35. Sainsbury A, Cusin I, Rohner-Jeanrenaud F, Jeanrenaud B. Adrenalectomy prevents the obesity syndrome produced by chronic central neuropeptide $\mathrm{Y}$ infusion in normal rats. Diabetes. 1997; 46: 209-14.

36. Kristensen P, Judge ME, Thim L, Ribel U, Christjansen KN, Wulff BS, et al. Hypothalamic CART is a new anorectic peptide regulated by leptin. Nature. 1998; 393: 72-6

37. Johnen H, Lin S, Kuffner T, Brown DA, Tsai VW, Bauskin AR, et al. Tumor-induced anorexia and weight loss are mediated by the TGF-beta superfamily cytokine MIC-1. Nat Med. 2007; 13: 1333-40.

38. Broberger C, Holmberg K, Kuhar MJ, Hokfelt T. Cocaine- and amphetamine-regulated transcript in the rat vagus nerve: A putative mediator of cholecystokinin-induced satiety. Proc Natl Acad Sci U S A. 1999; 96: 13506-11.

39. Wynne K, Stanley S, McGowan B, Bloom S. Appetite control. J Endocrinol. 2005; 184: 291-318.

40. Bi S, Kim YJ, Zheng F. Dorsomedial hypothalamic NPY and energy balance control. Neuropeptides. 2012; 46: 309-14.

41. Kesterson RA, Huszar D, Lynch CA, Simerly RB, Cone RD. Induction of neuropeptide $\mathrm{Y}$ gene expression in the dorsal medial hypothalamic 
nucleus in two models of the agouti obesity syndrome. Mol Endocrinol. 1997; 11: 630-7.

42. Lee SJ, Kirigiti M, Lindsley SR, Loche A, Madden CJ, Morrison SF, et al. Efferent projections of neuropeptide Y-expressing neurons of the dorsomedial hypothalamus in chronic hyperphagic models. J Comp Neurol. 2013; 521: 1891-914.

43. Acuna-Goycolea C, Tamamaki N, Yanagawa Y, Obata K, van den Pol AN. Mechanisms of neuropeptide $Y$, peptide YY, and pancreatic polypeptide inhibition of identified green fluorescent protein-expressing GABA neurons in the hypothalamic neuroendocrine arcuate nucleus. The Journal of neuroscience : the official journal of the Society for Neuroscience. 2005; 25: 7406-19. 